\title{
Sacrifice du corps et transmission dans Alma, Amédé et Prudent de Georgette Leblanc
}

\author{
Francis LANGEVIN, University of British Columbia (Okanagan)
}

Articuler le singulier au collectif, c'est rendre au collectif son peu de cohérence en même temps que lui donner un sens et une interprétation qui trouvent base et socle à l'intérieur des fragilités et des hésitations d'un destin commun, d'un monde qui ne cherche qu'à exister et qui n'existe que dans cette tension infinie entre ses désordres et ses contraires. Célébrer l'unité est une chose, la célébrer en sachant qu'elle est construite de chaos initiaux lui donne la dynamique même des chemins à parcourir. (Farge 6)

Cette lecture des corps dans Alma, Amédé et Prudent met en lumière des corps qui se transforment et qu'on dirait à première vue sacrifiés, car ils dévient de leur trajectoire initiale, une quête horizontale, c'est-à-dire géographique, spatiale, et c'est cet écart de trajectoire qui fait l'objet de chacun des recueils. Alma qui s'écarte de son envie de grouiller, Amédé qui s'éloigne du Village, Prudent contraint de courber l'échine et déporté. Mais chacun transforme ce corps afin de transmettre son essence: Alma devient cette maison solide pleine d'enfants, droite, «grande et solide astheure » $($ Alma, 112$)$; Amédé devient ce relief dans la cire qui pour toujours porte la mémoire du Village tel « le grand cyprès » (Amédé, 81); et Prudent devient libérateur en devenant le bateau qui emporte tout son monde et grâce à un acte de reconnexion, à la fois prière et rituel animiste : il arrête «d'être Prudent » ${ }^{1}$ et devient «l'Orignal», «le Trésor » (Prudent, 121). Je mettrai de côté pour l'instant le contexte d'émergence de cette œuvre, son positionnement dans la littérature acadienne, dans la littérature féminine ou même dans l'institution littéraire francophone, sauf pour marquer sa singularité. En effet, comme le remarque Catherine Leclerc, dès Alma, Georgette LeBlanc «s'écarte des voies modernisantes, urbaines, et contre-culturelles privilégiées par les poètes de Moncton depuis Gérald Leblanc » (59). En travaillant la « saveur de la langue de la Baie-Sainte-Marie » (Lonergan 32) alors que la tendance serait plutôt à la normalisation (38), ces recueils revisitent les exodes et les exils propres à l'imaginaire acadien, un «imaginaire des origines... [s'appuyant] sur une volonté de conciliation des contraires » (Boucher 154). Loin de cultiver une Acadie uniquement identitaire ou insulaire, et en marge du topos poétique de la ville au moyen duquel, écrit Jacques Paquin, les 
poètes acadiens de la génération précédente «apport[aient]... leur contribution au poème acadien de l'Amérique » et manifestaient une «nouvelle conscience continentale (13), l'œuvre de LeBlanc embrasse très largement le continent, de la Baie à la Louisiane et dépasse « l'archipel identitaire francophone sur l'océan Amérique » dont parle Pierre Nepveu². Je présente ici une lecture personnelle qui cherche à entrer en conversation, en résonnance avec les associations sémantiques, à la manière de la microlecture ${ }^{3}$. Très proche du texte, ma lecture sera attentive aux mouvements du corps des personnages dans Alma, Amédé et Prudent, trois recueils de la poète acadienne LeBlanc qui, en marge du renouveau monctonnien (Lord), urbain (Doyon-Gosselin), postmoderne (Boudreau), ou de «l'école d'Aberdeen » $\left(\right.$ Cormier) ${ }^{4}$, développent une singulière exploration de la vie de personnages référentiels de la petite et de la grande histoire acadienne. Ma lecture sera particulièrement attentive aux façons dont les corps, dans le paysage (Richard) et l'horizon (Collot) des poèmes comme dans leur texture (Samson) ${ }^{5}$, bougent, sentent, pensent, interagissent. $\mathrm{Au}$ moyen de récits biographiques intimes, familiaux et historiques en vers, LeBlanc raconte les métamorphoses du corps de personnages qui s'approchent du mythe identitaire en ce qu'ils se déroulent dans des ambiances de «temps primordial » (Eliade), une forme qui, comme l'écrit justement Leclerc (45-46), donne un sens différent au terme «écriture sauvage » mis de l'avant par Alain Masson.

\section{Danser}

\section{l'attitude c'est la différence entre la voix de ma mère et celle-là de la Vieille d'à côté (Alma, 18)}

Le pouvoir s'exerce directement sur les corps. Il y a une force centripète qu'exerce le sable des berges acadiennes qui enlisent Alma et la font prisonnière ; un fond chaud qui ne quitte jamais Amédé, même dans sa fuite, et une force qui empêche le Pembroke d'avancer vers le pays de la soumission. C'est ce balancier du corps, qui oscille entre le désir de désobéir à l'inertie c'est la danse, c'est la musique, c'est la fuite, c'est la mer, c'est la résistance aussi -, et la contrainte de marcher avec elle, de marcher sur place avec elle, de voir ses pieds s'enfoncer dans le sable du temps, progressivement, de prendre racine, car si le mouvement n'amasse rien, il ne vous débarrasse de rien non plus. C'est comme si les corps était la manifestation de séquelles ataviques. 
Alma, c'est d'abord la texture d'une peau dont « le corps nu menace » (9) et qui va s'habiller de brume. Dès l'ouverture du recueil, c'est la bouche (langue cachée dans les bois, petite goule, moue dans mourir, le sourire de la mère) et la voix d'Alma qui vont désigner son existence et les conflits qui lui donnent un relief dans la brume, mais c'est aussi sur les mains et les pieds que s'exerce le pouvoir temporel sous couvert de spiritualité, et qui va donner le piège de peau où Alma va se prendre les ailes. Pierrot va d'abord se manifester dans la langue, dans le sucre des mots à épeler dans la spelling bee. Ce désir d'être autre et ailleurs, d'échapper au sol et au corps atavique, féminin, maternel, c'est ce qui va à la fois perdre Alma et lui permettre de se trouver. Son émancipation, pour employer un grand mot du social, passera par l'expérience de la servitude.

Le corps d'Alma fut possédé et utilisé comme on colonise une terre, par oripeaux flamboyants de danse et de langue qui la font sortir de son intellect, de sa langue, de ses réflexes de survivance, pour l'emporter dans le goût de la grouille - de la danse et de la musique, des pieds et des jambes qui s'ouvrent comme les jupes du ciel du village d'Amédé. Le corps d'Alma, de son plein gré et obtenu sous serments, porte fruits entre les jambes, apporte du pain entre ses mains, de sorte que son corps produit sans cesse de la vie alors qu'elle se rapproche de la mort. Végétale et sauvage, vierge et enfant, elle est protégée semble-t-il par son imaginaire et sa rêverie, par sa naïveté et son ignorance du monde référentiel et historique; domestiquée, elle devient humaine, c'est-à-dire femme, elle devient un corps en chair happé par les contingences du réel, les bibis de sa chair et les factures à payer. Le langage et la danse, les beaux mots et la prestance de son homme revenu des Soutanes Noires - du Collège -, c'était un miel irrésistible dans lequel ses pieds qui aiment grouiller allaient pourtant s'engluer. L'immobilité tant nondésirée, c'est le silence de la maison endormie - pleine des corps pleins de vie des enfants qui dorment et du mari qui macère tandis que le pain lève.

Si l'on reprend tout dans l'ordre, on constate que la véritable tension, dans Alma, se trouve entre la résistance et l'abandon, entre la soumission et la rébellion; voir la Chine ou courber l'échine. Elle envie les tramps, leurs sacs pleins, «la magie noire et épaisse qui reste collée à la peau » des voyageurs (23), et avant tout leur mouvement. Car là, au village des mères et des grands-mères, il n'y a que larmes et maigreur contre quoi le rêve a plus d'emprise que les actions. La lecture permet alors de voir se matérialiser le temps ; Alma compte les livres comme bientôt elle comptera les bibis. Il en va ainsi d'autres gestes que l'on fait avec les mains, comme 
la râpure, geste quotidien qui s'accompagne d'un chant rituel - plurer pleurer -, l'échine pliée, soumission au rite et résistance en même temps, car ni les Anglais ni les Soutanes Noires n'en veulent. Se soumettre à cet atavisme, c'est paradoxalement résister à ce qui vient de l'extérieur : un catholicisme rigide (les Soutanes Noires) et un protestantisme assimilateur (les Anglais), qui s'accommodent tous deux assez bien semble-t-il de la bigoterie de l'autre.

Pierrot, c'est cet ailleurs ambulant qui fait miroiter à Alma le minéral avenir du voyage (mica, quartz), de la langue, des rêves. Alma s'y laisserait bien prendre, dit-elle : «ma main, mon cou / la cheville de mon pied » (30). Et en plein jour. C'est très attirant, c'est une musique qui fait tanguer, grouiller : «J'écoute avec tout mon corps / comme si toute ma vie j'avais su / que c'est de même qu'un corps devrait grouiller» (32). La danse, donc. Sur place. Un mouvement qui libère sans pourtant briser les liens de la filiation complètement, sans déraciner la mousse. À demi-mot, le désir aussi bouille tel le fudge, il faut brasser ; je pense en particulier à l'homme dont le regard, la nuit, l'invite dans sa chaleur (« peeping tom ») et fait fondre l'eau du lac qui «se donne la permission / de déborder» (37). Cet érotisme onirique est un rappel de l'indépendance du corps avant sa sociabilisation, car, si le corps a une mémoire, il n'a pas d'histoire ; il est sensation avant d'être idées. Ce printemps au poème se manifeste dans le corps des femmes amaigries par l'hiver: «Les femmes avont pus de hanches » (37). Et dans le corps d'Alma, les menstruations qui fragilisent son corps fait pour bouger, la mobilisent au soin de la « coupure qui se ferme point / qui reste rouverte et cachée / qu'il faut protéger / comme qu'on protège ceux-là qu'on aime du mal» (39), ce qui la rend bien distincte de Pierrot, encore davantage différente de l'homme qui «saigne rinque quand ce qu'il se coupe » (40). Au lieu de rester toute seule à «saigner dans [son] petit lit » (41), Alma et les autres jeunesses se rendent « communier » (43), façon détournée de dire en même temps la chose et son contraire, car tous deux se font bien avec un corps et avec une bouche. C'est pour danser, garçons et filles, qu'ils se retrouvent en cachette des Soutanes Noires, façon aussi pour Alma de se rapprocher du corps de Pierrot, des mains de Pierrot, «toute sa paume contre la sienne» (45). Ces rencontres en cachette racontent en creux à quel régime les corps sont contraints. Se marier, pour nos grands-mères, c'était se libérer.

Tout nous conduit, dans Alma, vers cette union des corps qui va rendre le pied léger, mousse et quartz «dans l'église de rosée » (49) - et jusque-là ça semble une bonne idée, car Pierrot part pour le Collège apprendre « la langue des Soutanes Noires » alors qu'Alma reste au 
logis pour apprendre, elle, « la langue de la misère » (49). La langue du pouvoir devrait donner à Pierrot une plus grande mobilité sociale, une plus grande liberté d'agir - et Alma n'aurait pas à se plier à un destin de servante. Il y a là un espoir. Mais ça ne fonctionnera pas ainsi. Quand Pierrot revient, il est asservi à un maître qui n'existe pas ; il a troqué son savoir culturel pour un autre, il ne peut plus «dire la différence / entre une pomme de pré pis une gadelle » (55). Cet effacement du patrimoine immatériel change aussi la langue et les manières de Pierrot. Si ce dernier a gardé la même bouche, les mêmes doigts, il semble avoir renoncé à ses « saisons » (56), aux savoirs ancestraux, expérientiels, corporels et temporels, à l'enfance, à la superstition, à la magie qui faisait tout son attrait. Sa servitude lui vient par là où elle aurait dû être effacée. Elle aurait dû lui permettre de nommer, donc de faire exister les mots pour nommer cette condition : servitude, esclavage.

Il n’y a pas vraiment de légèreté à attendre de ce côté, et voici 1929, la Crise et les exilés qui reviennent avec tout le poids de leur corps, « comme des bêtes » (59), expulsés par une ville, immense organe sans cœur. Autant s'aimer! Alma exprime comment, quand Pierrot « rouvre sa femme mariée » (63), elle reste silencieuse, sans voix, un corps sans langage sonore. C'est sous le signe de la danse, de la valse, que sera placé leur mariage qui commence par un corps-à-corps de fabrication du pain (68), geste sensuel et fécond s'il en est un, acte par excellence de l'alchimie des mélanges faits par les mains, consommation de la domesticité, moment de fondation d'un foyer: malaxage, humidité, fermentation, levage, enfournage, mie craquante, partage avec les doigts. Mais cette boulangerie érotique est tout juste suivie par un pas de recul de la danse, quand Pierrot dérape, «quand ce qu'il décolle sur ses trips» (69). Proximité des corps, éloignement des corps, rapprochement des corps, fuite, poursuite, divorce. Danse solo.

«[M]on corps est fait de lait et de racines » (71)

Comparés au lieu de la fabrication du pain, le four-ventre des mères et le rôle nourricier qu'Alma assigne à son corps la rendent progressivement moins libre pour la danse à mesure que ce corps devient maison; ces grossesses deviennent, selon la Soutane Noire, la façon de « débarrasser [ce] corps de terre et de mousse » (75). Leur maison est pourtant un sens unique, une cage à homards (76) pour Pierrot dont le corps change aussi (78), lui qui ne sort plus que pour la danse, ce qui est de plus en plus un prétexte pour toucher la bouteille... et pour voir Grace. Grace, femme à chapeaux qui «peut voler parce qu'elle est vide en dedans » (86), a sa liberté, et il est clair qu'Alma, ayant cru saisir la sienne avec Pierrot, en unissant son corps à 
celui de Pierrot, s'y est au contraire asservie en raison de ce corps, fertile pâte de mousse. Pierrot, corps sur le couch, vogue lentement vers nulle part en regardant la télévision. Les enfants, consolation ou misère, sont, suivant la métaphore boulangère, les pains qu'Alma fabrique de ses mains et avec son ventre et qu'elle décrit comme des morceaux, des prolongements de sa peau, mais aussi comme des pertes détachées, des soustractions à son entièreté : « une miette de vie déchirée de ma peau / une miette de moi-même pour couvrir cte petite bête-là » (93). Les multiples grossesses d'Alma, annuelles, ne sont pas qu'un produit des envies de Pierrot : elles sont le symptôme d'un système extérieur à eux qui rend Alma captive et qui confirme ainsi sa sédentarité atavique. Pas de Chine pour elle, pas de Chapeau, rêves abolis qui persistent dans une envie de voyager sans son corps : « ej vois mon envie de voler / comme si j'étais pu dans mon corps / comme si l'envie était un chapeau / sur la tête d'une autre femme / une femme perdue dans la brume » (95). Ce corps, qu'on a dit végétal, enraciné donc, où s'abreuve Pierrot, à sa sève, au candy, est puni, privé de plaisir parce que l'homme est allé goûter un autre lipstick. C'est une double pénitence quand Alma ferme les cuisses, « comme j'ai jamais gardé de-quoi de fermé de ma vie » (103). Le mari reste inactif sur le couch, et il n'y a plus qu'elle désormais pour garder les enfants. Alma s'achève avec cette révélation, qui survient un peu abruptement et sans motivation narrative nette : c'est en elle-même qu'Alma trouve son pas, «les pieds légers » (110), en mettant Pierrot à la porte de sa maison, où naît une «neuve façon de grouiller » (111). La matière - végétale - de son corps, terre épaisse aux senteurs de mousse, expulse le corps minéral de Pierrot, bouteille de vitre, corps étranger depuis toujours. $* * *$

\section{Grouiller}

《de parenté à parenté à parenté » (Amédé, 21)

Amédé y réussit mieux. À grouiller. Il s'apparente à Alma en raison de son goût de la danse, de l'énergie du voyage et d'une nature ambivalente de végétal pouvant absorber «le tourment du cri / toute la misère de retrouver la terre, l'encre du temps / de parenté à parenté à parenté » (Amédé, 21). Tout était déjà contenu en Alma: la grouille, l'envie d'échapper aux contingences, la rêverie sensorielle et l'imaginaire (le goût de l'Autre et de l'ailleurs). La voix d'Amédé, « le cri d'Amédé [...] dans la nuit / comme un voyage / comme un très long voyage / qui recommençait » (25) - contient le voyage des ancêtres. Cet atavisme du mouvement est, comme chez Alma, une tension, une indécision qui ne se résout que dans des circonstances 
brutales qui n'acceptent pas la valse-hésitation. Aimer Rose, femme de Grosse Tête, c'était pour Amédé un désir inaccessible comme la Chine : "Comment trouver une autre façon d'aimer / Rose» (32) ? Il n'y a pas d'autre moyen que de partir. Avant la tempête qui va dévaster le Village, survient le temps de la boucherie, où le corps d'un cochon est divisé - « gros corps après se faire trancher, du nord au sud» (40). Amédé sent «l'envie braquer» (42). Résolution bien corporelle, physique d'un problème éthéré, l'attirance, le désir : Amédé se résout à partir pour faire chanter sa Cordine et «chaque chambre de son poumon bienaimé » (54). Amédé procurera le souffle qui manque à Lejeune, son partenaire de jeu, violoniste au cœur brisé par la noyade de Jolie Brune. Leur équipée est soudée par la catastrophe de la noyade, de la tempête qui inonde le cœur du village et que ni le violon de Lejeune ni l'accordéon d'Amédé, leur tune, ne réussissent à empêcher de couler. Si le village a un cœur, la tempête, elle, a des jambes de femme qui veut danser :

Amédé aurait pu dire à cte moment-là

il aurait pu chanter que c'était une grande déchirure

qu'il avait entendu la skirt du ciel se défaire

chaque fibre, chaque point de travail

comme si le corps qu'i l'avait habitée était rinque venu trop plein

trop plein de vie, trop plein de tout ça qui guette

comme s'il y avait plusse de place dans le ciel (45)

Le départ de Lejeune et d'Amédé vers le grand Texas «dans le ventre de la train » $(55 ; 57)$, c'est à mon sens une double exacerbation des opposés du Village. D'abord l'imaginaire du sec des vachers et du désert, qui s'opposent clairement au cataclysme où se sont mêlées les eaux du bayou, du fleuve Mississippi, de la rivière Rouge, et les eaux salées montantes de la mer qui «avait ramené le voyage, le besoin de grouiller » (51). L'Atchafalaya en déroute, raconté comme un naufrage, c'est aussi une remémoration nourrie à un imaginaire de l'exode, celui des botes, de la navigation, une pratique qui renvoie au passé, aux attachements, aux détachements, à l'isolement et enfin à une idéologie de la conservation, de la filiation mémorielle où le temps s'arrête. Coupés du Nouveau Monde, coupés de l'Ancien Monde. Autarcie craintive. Le Texas, deuxième opposition, c'est l'Amérique décomplexée de la connexion, le regard posé sur un avenir à construire, comme les États d'avant la Crise évoqués dans Alma, mais cette fois en glorieuse expansion. L'exil de Lejeune et d'Amédé commence sur la train, véhicule par excellence de la connectivité continentale : 
au Grand Texas

à galoper le triangle, l'industrie des chemins pavés

dans tout ça qui roulait et qui voulait rouler

dans la poussière qui mirait, qui craquait d'électricité

[...]

au Grand Texas, le carré du Village avait été remplacé

la musique sortait pus de la terre

de la sueur d'une semaine de travail

la musique sortait de l'électricité

et pouvait sortir partout en même temps

pouvait allumer la nuit comme le jour (58)

Désormais musiciens de profession, les deux hommes font danser les villes en puisant dans le corpus du Village. Le recueil raconte que leurs chemins se séparent quand Lejeune s'éloigne, « la lumière au bras », c'est-à-dire une femme, Jolie Blonde (61). Amédé continue à jouer, « son grand corps seul, la tête dans les nuages / dans les pétales de Rose» (61); il va rencontrer Savoie, qui souhaite enregistrer les histoires que peut chanter la Cordine d'Amédé. C'est à ce moment, me semble-t-il, que son histoire s'apparente à la découverte que fait Alma, et elle vient en quelque sorte l'éclairer :

ça fit drôle à Amédé de l'entendre parler de l'entendre dire ça qui avait rinque pensé

dans la quête de Savoie, d'un Étranger, Amédé comprit que ça que depuis toute une vie Amédé avait cherché

que ça que depuis toute une vie Amédé avait senti

comme un vide

que tout ça était peut-être là, en lui

tout le Livre du Village, le cyprès, le corps et la famille étaient en lui (65-66)

La corporéité de la mémoire, qui pesait tant à Alma, est ici source de réconfort. Apprenant de surcroît le même soir que Rose s'est elle aussi exilée, Amédé écrit des chansons qu'il enregistre pour Savoie; il les joue « comme on grouille pour se retrouver » (68). Plusieurs années semblent se passer avant que Lejeune et lui se retrouvent à la Nouvelle-Orléans, dans la «Ville Plate » en plein Mardi Gras pour recréer « le Village dans la Ville » (75), un duo du poumon de la Cordine d'Amédé et des coups d'archet de Lejeune. De l'individu blessé, en fuite, en marge, à la collectivité en réparation, il y a l'espace d'un cri, « un cri chaud, épais » (76), que leur musique va lancer. Ce cri, autrement physique, ramasse les différentes trames thématiques développées dans Amédé et dans Alma : le végétal, la terre, le mouvement, l'eau, le corps, la mémoire - que l'on peut résumer par ce vers : «après tant d'années à nager enraciné » (76). Après avoir porté 
les histoires du Village en lui, «le Livre dans le ventre », après les avoir sorties de lui et transmises, Amédé soustrait son propre corps en se jetant sous «le fer chaud de la car», «sous la pesanteur de la roue », « la roue au travers de son corps » (78-79). Suicide, démantèlement du corps en symétrie avec le corps découpé du cochon au Village la veille du déluge : tout se passe comme si Amédé n'avait été que l'intercesseur, une sorte de traducteur des histoires du village, son corps recueillant le Livre pour le sauver du naufrage, en faire une trace dans la cire, «les sillons du cercle, la mer de la grande plaine salée » (81). C'est en quelque sorte une histoire christique, à cause du sacrifice de la vie temporelle d'abord, mais aussi à cause de l'incorporation de ce qui pourrait bien être l'âme du Village, le cri de la «parenté perdue » (81) qui permet au Village, par la musique, mémoire immatérielle matérialisée, de se refaire. La musique devient un rituel semblable à une prière qui peut être répétée tant dans le ventre de la hall que sur les carrés de village.

\section{Danser. Grouiller. Résister.}

\section{Il faut le prendre! Il faut prendre le bote! Il faut reprendre nos corps! (Prudent, 105)}

Prudent et 1'Eucharistie. J'écris aussi Prudent et la Communion. Je ne sais pas quoi faire de ces christianités répétées. Tous ces personnages de phœnix qui s'effacent, brûlent et renaissent (transformés, ceci dit) pour le bénéfice des autres. Autant Alma qu'Amédé prennent des décisions qui sont bien individuelles, et que ma lecture a cherché peut-être à allégoriser vers le collectif, le genre féminin, la génération, la race, la marginalité. Mais ce sont surtout des projets amoureux, liés au désir et au corps, qui les placent dans les situations inconfortables qui seront le plus bas de leur parcours - et dont ils vont tous deux se sortir en changeant de corps : la première, mariée et malheureuse, étend son corps à la dimension de sa maison; le second, exilé solitaire un temps porte-musique, devient une gravure dans un sillon de cire et un fantôme des musiciens. Mais avant le corps d'Alma dans la Crise de 1929 et le corps d'Amédé après le déluge de l'Atchafalaya (1927), voici la voix de Prudent l'Ancien (1755), cet intermédiaire entre le pouvoir des sans-mots et la faiblesse muette ; Prudent porte-parole, celui-là, d'une collectivité captive en plein cœur du Grand Dérangement. À la croisée des cultures Mi'kmaq, française et anglaise, il porte de nombreuses loyautés cumulées dans son grand âge, loyautés qui devaient, c'était son projet, amener ou conserver la paix. Prudent avait intellectualisé la paix, l'avait crue 
possible dans le langage, hors des corps et des armées : il sera «embarqué Comme du bétail » (Prudent, 13), et ramené à sa somme d'os, de muscles, de sueur, de soif et de faim. Comme la Crise et comme le cataclysme, la Déportation, geste on ne peut plus corporel, amène Prudent dans le ventre d'un bateau anglais : le Pembroke. Prudent Robichaud, né à Port-Royal en 1669, mort à Rivière-Saint-Jean à l'été 1756, six mois après la légendaire mutinerie de prisonniers acadiens qui, à bord du Pembroke, saisissent l'équipage de huit Anglais et détournent le bateau à Sainte-Anne-des Pays-Bas. Prudent, c'est l'histoire de cette reprise en main des corps à la dérive, un sauvetage aux proportions de légende. Trente-deux familles déportées le jour de l'Immaculée Conception de 1755. Trente-deux familles, c'est deux cent trente-deux personnes. Une partie seulement de la famille de Prudent Robichaud, dit l'Ancien. Le Pembroke qui, selon les sources historiques consultées ${ }^{6}$, est repris par six Acadiens sachant naviguer et ramené à l'embouchure du Fleuve Saint-Jean le 8 janvier 1756. L'aspect légendaire de l'histoire tient au déséquilibre entre les forces anglaises (huit hommes) et sa cargaison (deux cent-trente-deux Acadiens, dont des marins expérimentés). Prudent va prendre la parole : la moitié du recueil, en italiques, est composée de cette prière qui va naître en l'Aîné pour parler de la soumission et de la misère souffertes sans espoir de réussite :

Jusque là, à se coucher parmi les rats morts.

À marcher dans le noir, à marcher l'échine courbée.

À fouiller dans la misère pour arrêter le petit bibi de brailler.

À couler, longis, dans l'eau trouble de la fin, dans la cale comme l'intestin.

À guetter les restes d'la pluie morte à tomber, à guetter les petites lueurs de lumière noyées. Jusqu'à perdre l'envie de croire au jour. (26)

Prudent va raconter l'histoire, qui va de son «échine droite » (27) à son «échine courbée » (26). Cette histoire individuelle appelle une lecture métonymique, où Prudent est cet homme au corps adapté au territoire qu'il dit avoir tracé (27): "J'avions toutes la terre d'arpentée dans nos corps, / dans notre mémoire », dit-il en prière (45). Il a trappé le castor pour mettre «des chapeaux sur la tête du Roi » (28), il n'y a aucune «Robe Noire dans la cale » (29), et les Red Coats les maintiennent captifs d'un navire qui les déporte. Prudent l'Aîné est le porteur vivant d'une mémoire des corps qui ont cherché à créer un Nouveau Monde métissé qui, au moment de la prière, leur est refusé. Le début de cette prière raconte une chasse à l'orignal initiatique qui va permettre à Prudent de «prendre femme et famille » et de consacrer son union avec Henriette 
Petitpas, «[b]rune comme la terre » (30). La mise à mort de l'animal est une incorporation rituelle où Prudent disparaît une première fois :

C'était mon tour.

Ej fis comme j'avais rêvé

Ej laissis la bête rentrer, ej rouvris mon corps comme un port

Ej tiris l'arc, ej hâlis fort.

J'emmenis son regard dans le mien.

Ej laissais aller. Nos cours percés.

C'était mon tour. (33)

Prudent raconte ce refus d'être pionnier, de céder à cette mentalité du settler qui conduit à mettre deux cent trente-deux personnes dans la cale d'un bateau anglais en plein décembre. Il était davantage intéressé à écouter qu'à parler, à apprendre qu'à enseigner :

Pour écouter, il fallait se taiser.

Fallait suspendre ses racines, fondations, foyers

pour faire de la place pour l'autre,

pour mieux voir ça que l'autre voulait nous montrer. (43)

Plus tard,

Prudent parlait de faire la Paix comme une façon de grouiller.

S'il pouvait rinque arriver à écouter fort assez.

Faire assez de place dans sa tête et son cœur,

pour tout le monde,

ça pourrait se régler.

Il y avait de la place en masse.

Ça prenait un espace pour voir dans les yeux de l'autre,

la Bête, l'Infortuné. (45)

Prudent avait créé cet espace pour la rencontre : «Red Coats, Blue Coats, Robes Noires et Plumés / pouviont entrer et sortir, / notre logis était un havre sacré par le travail, par l'entente. » (50). Alors que Prudent raconte son désaccord avec son fils Joseph, sans doute la partie la plus narrative du recueil, le vent a tombé et le Pembroke n'avance plus. Joseph le «batailleur » (74) avait été chassé de la maison; il parlait haut et bien de la servitude et la refusait ; il avait souhaité «reprendre le paradis» (55). Alors que la masse des deux cent trentedeux passagers fait corps avec le navire grâce à cette prière qui prend la forme d'une méditation sur la vie et la mort (donner la vie, prendre la vie), c'est le pardon, la rédemption, puis enfin la réparation de la filiation brisée par un fils et son père qui vont redonner à ces corps captifs l'énergie de leur masse. Joseph avait mis en danger l'idée de paix de Prudent l'Ancien, il avait donné de la voix. 
À ce moment où le bateau est toujours immobile, Prudent regrette de ne pas avoir écouté Joseph, car le plan de paix avait échoué : le commandant Handfield allait venir avec des ordres et emprisonner Prudent «qui bâtissait les ponts » (73). Désillusion, trahison. «Prudent avait point écouté, mais il était là asteure. / Prudent avait son fils à ses côtés, retrouvé » (76), reprend le récit. Le vent aussi revient, et « les côtes de la cale » (80) sont gonflées de la rage de Prudent ; un chœur va animer d'un souffle le ventre du bote jusqu'à ce qu'une tempête au-dehors vienne confirmer la force de la complainte de l'Aîné. L'introspection de Prudent avait arrêté le bateau de déportation des vaincus résignés ; l'aveu de Prudent avait relancé les vents ; sa rédemption avait redonné une voix puissante à Prudent mais aussi à la collectivité (qui d'ailleurs n'est qu'une seule voix, elle est chœur et unie). Fin de la résignation, fin du silence pour «les corps gaspillés et vendus pour le marché » (83) tel que l'aurait souhaité Joseph. Le territoire, la marée, la nuit, les étoiles, le ciel, les éloizes et la Baie conspirent pour défaire les calculs du Capitaine ; et tandis que la tempête gronde, la prière monte ( «Et tout le monde en sortait les épaules relevées » [98]), et bientôt les hommes forts sortiront de la cale et iront «monter l'escalier. / La porte s'ouvrira une fois pour toutes » (103) : «La pression, le feu des paroles de l'Ainé aviont réussi. » (107) C'est ici son corps, encore une fois, et l'expérience ou la mémoire qu'il contient, qui permet un retour au Paradis comme le retour du Livre du Village d'Amédé :

Il avait été vidé

Son corps vieux de ses quatre-vingt-six ans braquit à trembler, à trembler comme une femme qui vient d'accoucher.

L'Ainé avait prié avec tout ce qu'il avait de reste en lui.

L'Ainé avait offert son cœur, son sang, ses tripes aux autres. (112)

Tout cela est bien littéral, j'en conviens (« cœur », « sang », «tripes »), et contribue à nourrir la figure du corps sacrifié dont je viens de parler à propos d'Alma et d'Amédé, métonymie de la résistance du corps temporel et d'un certain imaginaire (chrétien) de l'âme. En plus du fils retrouvé, le récit va jusqu'à expliciter cette trajectoire de corps à corps, de la main du père à la main du fils, et proposer une allégorie d'une partie de l'identité diasporique acadienne: «Prudent avait rebraqué le feu de sa lignée. / Prudent avait repris la main de son fils. / En prenant le bote, il aviont repris la boussole. / Il retrouverait son petit-fils. » (113).

La dernière partie du récit de Prudent file plus loin cette analogie entre le corps de la lignée de l'Aîné et le territoire, analogie qui avait été aperçue dans la cuisine d'Alma et dans les racines d'Amédé. Une dernière prière, en quelque sorte la mort de Prudent, raconte son retour à 
l'orignal pour devenir cet animal, pour perdre son corps et transmettre son idéal de paix en fusionnant avec ce qui était là bien avant : «La Bête grande comme si elle prenait tout le ciel, / le muscle, la peau shinée, le soleil frette de l'automne à faire briller. / Le corps, le sang, le fruit du Temps » (118).

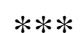

J'avais souhaité parler de la politique des corps chez LeBlanc. Je pensais en particulier au corps d'Alma elle-même, personnage féminin inaugural de l'œuvre de l'écrivaine ; je pensais à l'aspect collectif des corps, corps métonymique de la diaspora dont les actions individuelles auraient été la métonymie d'une résistance collective. Le motif que j'ai plutôt trouvé, comporte bien une dimension de résistance à des forces collectives étrangères à la nature de ces corps (genre, race), mais c'est avant tout en sacrifiant ces corps, le leur, qu'Alma, Amédé et Prudent permettent l'acte de résistance le plus important : la transmission. Trois corps qui se détachent de leurs relations horizontales et qui creusent ou érigent des relations verticales ; trois corps qui s'effacent en retrouvant «l'épaisseur de la terre» (Alma, 112), les pieds «bien plantés, enracinés » (Amédé, 81) et qui se remplissent de territoire (Prudent, 11).

\section{Bibliographie}

Boucher, Monique. «Exil et exode : l'imaginaire des origines ou origines de l'imaginaire ». Dir. James Finney, Hélène Destrempes et Jean Morency. L'Acadie des origines: mythes et figurations d'un parcours littéraire et historique. Sudbury : Prise de parole, 2011. 143-154. Eliade, Mircea. Aspects du mythe. Paris : Gallimard (Folio/essais 100), 1963.

Farge, Arlette. «Corps et politique : individu et société ». French Politics, Culture \& Society (2006) : 1-7.

LeBlanc, Georgette. Alma. Moncton : Perce-Neige, 2006.

---. Amédé. Moncton : Perce-Neige, 2010.

---. Prudent. Moncton : Perce-Neige, 2013.

Leclerc, Catherine. «"Écriture sauvage," tradition, et renouvellement en poésie acadienne ». Quebec Studies 43 (2007) : 43-66.

Lonergan, David, dir. Paroles d'Acadie : anthologie de la littérature acadienne, 1958-2009. Sudbury : Prise de parole, 2010.

Nepveu, Pierre. 
Paquin, Jacques, dir. Nouveaux territoires de la poésie francophone au Canada 1970-2000. Ottawa : PU d'Ottawa, 2012.

\section{NOTES}

${ }^{1}$ Les italiques dans toutes les citations des œuvres de LeBlanc sont de l'auteure.

${ }^{2}$ Je renvoie ici à l'article de Pierre Nepveu « L'océan Amérique : notes sur un archipel identitaire » (dir. Lélia L. M. Young, Langages poétiques et poésie francophone en Amérique du Nord, Québec : PU Laval, 2012. 17-30).

${ }^{3}$ Voir par exemple Microlectures de Jean-Pierre Richard (Paris : Seuil [Poétique], 1979).

${ }^{4}$ Je fais allusion aux travaux effectués, dans Écrire au féminin au Canada français (dir. Johanne Melançon Sudbury : Prise de parole, 2013), par Marie-Linda Lord ( « L'“arrivée en ville” de l'avenir féminin de l'Acadie dans Les confessions de Jeanne de Valois d'Antonine Maillet», 49-63) et par Benoit Doyon-Gosselin ( Le tournant spatio-référentiel dans l'œuvre romanesque de France Daigle», 65-82), ainsi que, dans Itinéraires de la poésie: enjeux actuels en Acadie, en Ontario et dans l'Ouest canadien (dir. Robert Yergeau, Sudbury: Prise de parole, 2004), par Raoul Boudreau («La poésie acadienne depuis 1990 : diversité, exiguïté et légitimité », 83-97) et, dans Paquin, par Pénélope Cormier («Les jeunes poètes acadiens à l'École Aberdeen: portrait institutionnel et littéraire », 179-204).

${ }^{5}$ Ma réflexion s'inspire des travaux de Jean-Pierre Richard dans Microlectures, mais également de ceux de Michel Collot dans L'horizon fabuleux (Paris : Corti, 1988), et d'Hélène Samson dans Le tissu poétique de Philippe Jaccottet (Bruxelles : Mardaga, 2004).

${ }^{6}$ Voir Placide Gaudet, «Récits relatant la prise du Pembroke par les Acadiens, 1755-1756» (Bulletin des recherches historiques, 14.1 (1908) : 41-54). 